\title{
On the Wintner-Ingham-Segal summability method
}

\author{
S. Kanemitsu, T. Kuzumaki and Y. Tanigawa
}

To the memory of Professor S. Srinivasan, with friendship and respect

\begin{abstract}
The aim of this note is to establish a subclass of $\mathcal{F}$ considered by Segal if functions for which the Ingham-Wintner summability implies $F$-summability as wide as possible. The subclass is subject to the estimate for the error term of the prime number theorem. We shall make good use of Stieltjes integration which elucidates previous results obtained by Segal.

Keywords. (Ingham) summability, zero-free region, Stieltjes integral, weak Riemann hypothesis

2010 Mathematics Subject Classification. 40D99, 11M99
\end{abstract}

\section{Introduction}

The purpose of this note is to establish a subclass of $\mathcal{F}$ for which $I$-summability implies $F$-summability as wide as possible, the subclass being subject to the estimate for the error term of the prime number theorem, PNT. It will turn out that such a result depends on a delicate relation between the reducing factor appearing in (1.3) and in the estimate for $\mathrm{M}(x)$. We may slightly improve the result of Segal by appealing to the best known estimate (1.6) for the Möbius functioncolorred, the result being stated in Theorem 3.4. Seondly, we shall elucidate the argument in [Se75] by incorporating the view point of the Stieltjes integration.

Given a sequence $\left\{a_{n}\right\}$ let for $x \geq 1$

$$
I(x)=\frac{1}{x} \sum_{n \leq x} \sum_{d \mid n} d a_{d}=\sum_{d \leq x} a_{d} \frac{d}{x}\left[\frac{x}{d}\right],
$$

where $[x]$ denotes the integral part of $x$. We extend the domain of $I$ by assuming $I(x)=0$ for $0<x<1$,

The series $\sum_{n=1}^{\infty} a_{n}$ is said to be $I$-summable to $A$ if

$$
\lim _{x \rightarrow \infty} I(x)=A .
$$

Or more precisely, Wintner-Ingham summable. [Win43], [In45], [Har49], [Win57]

Definition 1. The class $\mathcal{F}$ consists of all $F:[0,1] \rightarrow \mathbb{R}$ of class $C^{2}$ which are monotone nonincreasing with $F(0)=1$ and $F(1)=0$, The domain is extended by assuming $F(x)=0$ for $x>1$. Further $-F^{\prime}$ is monotone non-increasing and the order condition is satisifed:

$$
F\left(1-\frac{1}{v}\right)=O(\delta(v)), \quad v \rightarrow \infty
$$

where $\delta(v)$ is the reducing factor related to the PNT and

$$
\delta(v)=\delta_{c}(v)=e^{-c(\log v)^{3 / 5-\epsilon}}
$$

for every $\epsilon>0$ unconditionally, $c$ not being the same at each occurence and

$$
\delta_{R H}(v)=e^{-\epsilon(\log v)},
$$

We thank episciences.org for providing open access hosting of the electronic journal Hardy-Ramanujan Journal 
on the weak $R H$ (in which case $0<c<\frac{1}{2}$ ). The delta functions are slowly varying functions which makes the estimation simpler, [deH70], [Sen76] The series $\sum a_{n}$ is said to be F-summable to $A$ if $\lim _{r \rightarrow \infty} A_{F}(r)=A$, where $A_{F}(r)$ is defined by (3.18).

It seems that Segal was led to the notion of $F$-summability by the following observation. The weight in (1.1) is of the form $v\left(\frac{1}{v}-\left\{\frac{1}{v}\right\}\right)=1-v\left\{\frac{1}{v}\right\}$, which he replaces by $(1-v)^{\delta}$ in order to accommodate the Riesz sum weight (Cf. [CM52], [HR72]). In this case $F\left(1-\frac{1}{v}\right) \sim\left(\frac{1}{v}\right)^{\delta}$, which is his original definition. It turns out that one may assume a looser condition if one appeals to the best known bound for the error term in PNT.

For the arithmetical function $h$ (which may be either the Möbius function or the one defined in (3.25)) we also assume the estimate in relation to (1.3):

Assumption.

$$
\mathrm{M}(x)=\mathrm{M}_{h}(x)=\sum_{n \leq x} h(n)=O(x \delta(x))
$$

and

$$
h(n)=O\left((\log n)^{A}\right)
$$

for an arbitrarily large $A$ unconditionally and

$$
\mathrm{M}(x)=\mathrm{M}_{h}(x)=O\left(x^{b}\right), 0<b<1
$$

on the weak RH (WRH). Here the weak RH means that there exists an $a>\frac{1}{2}$ such that $\zeta(s) \neq 0$ in $a \leq \sigma \leq 1$. This implies for any $a<b<1, \sum_{n \leq x} \mu(n)=O\left(x^{b}\right)$. For the error term of the PNT, cf. [Da80], [Iv85] [Tit86].

Table Reducing factor and $M_{h}$ estimate

\begin{tabular}{|r|l|c|}
\hline cond. & red. factor & $M_{h}$ estimate \\
\hline uncond & $(1.4)$ & $(1.6)+(1.7)$ \\
\hline WRH & $(1.5)$ & $(1.8)$ \\
\hline
\end{tabular}

\section{Preliminary materials}

All the tools we need are contained in

Theorem 2.1. - (i) The Stieltjes integral exists if $f$ is continuous and $g$ is of bounded variation. The role can be changed in view of Item (ii).

- (ii) The formula for integration by parts holds true:

$$
\int_{a}^{b} f(x) \mathrm{d} g(x)=[f(x) g(x)]_{a}^{b}-\int_{a}^{b} g(x) \mathrm{d} f(x),
$$

provided that $f$ is continuous and $g$ is of bounded variation or $g$ is continuous and $f$ is of bounded variation.

- (iii) If $g$ is a step function with jumps $a_{n}$ at $x_{n}$, the Stieltjes integral reduces to the sum:

$$
\int_{a}^{b} f(x) \mathrm{d} g(x)=\sum_{a<x_{n} \leq b} f\left(x_{n}\right) a_{n} .
$$


- (iv) If $f$ is continuous and $g$ is differentiable, then the Stieltjes integral reduces to the Riemann integral:

$$
\int_{a}^{b} f(x) \mathrm{d} g(x)=\int_{a}^{b} f(x) g^{\prime}(x) \mathrm{d} x .
$$

Remark 2.2. There are many instances where the partial summation and integration by parts are regarded as different processes. However, from the general point of view of Stieltjes integration they are exactly the same as shown below.

If in (2.10), $f$ is differentiable, then applying (2.9) to it, we deduce that

$$
\sum_{a<x_{n} \leq b} f\left(x_{n}\right) a_{n}=\int_{a}^{b} f(x) \mathrm{d} g(x)=[f(x) g(x)]_{a}^{b}-\int_{a}^{b} g(x) f^{\prime}(x) \mathrm{d} x,
$$

which is the partial summation. When $f$ and $g$ are differentiable of class $C^{1}$, say, we combine (2.9) and (2.11) to deduce

$$
\int_{a}^{b} f(x) g^{\prime}(x) \mathrm{d} x=[f(x) g(x)]_{a}^{b}-\int_{a}^{b} g(x) f^{\prime}(x) \mathrm{d} x,
$$

which is the formula for integration by parts.

For more details cf. e.g. [Ap57], [Wi89].

We shall make a good use of a continuous version of the Möbius inversion formula. We state a general form of the formula for curiosity. Cf. e.g. [HR72].

Lemma 2.3. (Möbius inversion formula) For arithmetic functions $f, g$, the following are equivalent $(n \in \mathbb{N})$ :

(i) $\sum_{d \mid n} f(d)=g(n)$;

(ii) $f(n)=\sum_{d \mid n} \mu(d) g\left(\frac{n}{d}\right)$;

(Continuous version of (i),(ii))

(i) $)^{\prime} g(x)=\sum_{n \leq x} f\left(\frac{x}{n}\right)$;

(ii) $)^{\prime} f(x)=\sum_{d \leq x} \mu(d) g\left(\frac{x}{d}\right)$;

(iii) $\sum_{n \leq x} g(n)=\sum_{d \leq x} f(d)\left[\frac{x}{d}\right], \quad 1 \leq x \in \mathbb{R}$,

where $[x]$ indicates the greatest integer $\leq x$.

Proof. We give a proof of (ii) ${ }^{\prime}$ which depends on the "double to repeated" integration principle. For arbitrary arithmetic functions $a, c$ let

$$
b(x)=\sum_{n \leq x} c\left(\frac{x}{n}\right) .
$$

Then consider the continuous convolution $a \tilde{*} b$ :

$$
a \tilde{*} b(x)=\sum_{n \leq x} a(n) b\left(\frac{x}{n}\right) .
$$


Substituting from (2.14), we deduce that

$$
a \tilde{*} b(x)=\sum_{d \leq x} a(d) \sum_{\delta \leq x / d} c\left(\frac{x / d}{\delta}\right)=\sum_{d \delta \leq x} a(d) c\left(\frac{x}{d \delta}\right)=\sum_{n \leq x} c\left(\frac{x}{n}\right) \sum_{d \mid n} a(d) .
$$

Putting $c(n)=1$ and $a=f$ in (2.16) gives (iii) in view of $b(x)=[x]$ while $a=\mu$ and $b=g$ gives (ii) $^{\prime}$ in view of

$$
\sum_{d \mid n} \mu(d)= \begin{cases}1 & n=1 \\ 0 & n>1\end{cases}
$$

\section{Theorem 3.4}

Our aim in this section is to prove Theorem 3.4 which entails the result that $I$-summability implies $F$-symmability for $F \in \mathcal{F}$.

We write $A(x)=\sum_{n \leq x} a_{n}$. Inverting (1.1) by Möbius inversion, Lemma 2.3, (ii) ${ }^{\prime}$, we have

$$
\sum_{d \leq x} \frac{\mu(d)}{d} I\left(\frac{x}{d}\right)=\frac{1}{x} \sum_{n \leq x} n a_{n}=\frac{1}{x} \int_{1}^{x} t \mathrm{~d} A(t)=\frac{1}{x}\left(x A(x)-\int_{1}^{x} A(t) \mathrm{d} t\right)
$$

by Stieltjes integration. Also by Stieltjes integration

$$
\sum_{k \leq r} a_{k} F\left(\frac{k}{r}\right)=\int_{1}^{r} F\left(\frac{t}{r}\right) \mathrm{d} A(t)=-\frac{1}{r} \int_{1}^{r} F^{\prime}\left(\frac{t}{r}\right) A(t) \mathrm{d} t .
$$

The left-hand side member is the $A_{F}(r)$ sum defined in (3.18).

Lemma 3.1. For every $F$ in the class $\mathcal{F}$ we have

$$
A_{F}(r):=\sum_{k \leq r} a_{k} F\left(\frac{k}{r}\right)=\sum_{d \leq r} \frac{\mu(d)}{d} \int_{1}^{r} \frac{I\left(\frac{t}{d}\right)}{t} G\left(\frac{t}{r}\right) \mathrm{d} t
$$

where

$$
G(x)=F(x)-x F^{\prime}(x)
$$

This is $[$ Se75, (4)] and its deduction is the simplest if we calculate the integral

$$
I=\int_{1}^{r} F^{\prime}\left(\frac{t}{r}\right) \frac{1}{t} \int_{1}^{t} A(u) \mathrm{d} u \mathrm{~d} t .
$$

in two different ways. One is as given by Segal and applying integration by parts as follows.

$$
I=\left[r F\left(\frac{t}{r}\right) \frac{1}{t} \int_{1}^{t} A(u) \mathrm{d} u\right]_{1}^{r}-r \int_{1}^{r} F\left(\frac{t}{r}\right)\left(\frac{1}{t} \int_{1}^{t} A(u) \mathrm{d} u\right)^{\prime} \mathrm{d} t .
$$

Noting that the first term of the right-hand side is equal to 0 and the derivative inside the integral is

$$
\frac{1}{t}\left(A(t)-\frac{1}{t} \int_{1}^{t} A(u) \mathrm{d} u\right)=\frac{1}{t} \sum_{d \leq t} \frac{\mu(d)}{d} I\left(\frac{t}{d}\right)
$$

by ([Se75, (1)], we obtain

$$
I=-r \int_{1}^{r} \sum_{d \leq t} \frac{\mu(d)}{d} I\left(\frac{t}{d}\right) \frac{1}{t} F\left(\frac{t}{r}\right) \mathrm{d} t=-r \sum_{d \leq r} \frac{\mu(d)}{d} \int_{1}^{r} \frac{I\left(\frac{t}{d}\right)}{t} F\left(\frac{t}{r}\right) \mathrm{d} t
$$


on noting that $I\left(\frac{t}{d}\right)=0$ for $d>t$.

The other way is to substitute for $\frac{1}{t} \int_{1}^{t} A(u) \mathrm{d} u$ from ([Se75, (1)]:

$$
I=\int_{1}^{r} F^{\prime}\left(\frac{t}{r}\right) A(t) \mathrm{d} t-\int_{1}^{r} \sum_{d \leq t} \frac{\mu(d)}{d} I\left(\frac{t}{d}\right) F^{\prime}\left(\frac{t}{r}\right) \mathrm{d} t .
$$

By $[\operatorname{Se} 75,(2)]$, the first integral is $-r \sum_{k \leq r} a_{k} F\left(\frac{k}{r}\right)$, so that it follows from (3.22) and (3.23) that

$$
\sum_{d \leq r} \frac{\mu(d)}{d} \int_{1}^{r} \frac{I\left(\frac{t}{d}\right)}{t} F\left(\frac{t}{r}\right) \mathrm{d} t=\sum_{k \leq r} a_{k} F\left(\frac{k}{r}\right)+\sum_{d \leq r} \frac{\mu(d)}{d} \int_{1}^{r} \frac{I\left(\frac{t}{d}\right)}{t} \frac{t}{r} F^{\prime}\left(\frac{t}{r}\right) \mathrm{d} t,
$$

or (3.18).

Proposition 3.2. For any arithmetic function $g(n)$ let

$$
b_{n}=\frac{1}{n} \sum_{d \mid n} d a_{d} g\left(\frac{n}{d}\right), \quad h(n)=\sum_{d \mid n} \mu(d) g\left(\frac{n}{d}\right) .
$$

Then we have

$$
B_{F}(r):=\sum_{k \leq r} b_{k} F\left(\frac{k}{r}\right)=\sum_{d \leq r} \frac{h(d)}{d} \int_{1}^{r} \frac{I\left(\frac{t}{d}\right)}{t} G\left(\frac{t}{r}\right) \mathrm{d} t
$$

which is a generalization of Lemma 3.1 with $g$ the multiplicative identity. This further leads to

$$
B_{F}(r)=\int_{1}^{r} \frac{I\left(\frac{r}{w}\right)}{w} H_{h}(w) \mathrm{d} w
$$

where

$$
H(w)=H_{h}(w)=\sum_{n \leq w} \frac{h(n)}{n} G\left(\frac{n}{w}\right)
$$

Proof. Substituting from (3.25), we deduce that

$$
\begin{aligned}
& B_{F}(r)=\sum_{k \leq r} F\left(\frac{k}{r}\right) \frac{1}{k} \sum_{d \mid k} d a_{d} g\left(\frac{k}{d}\right)=\sum_{d \delta \leq r} F\left(\frac{d \delta}{r}\right) \frac{1}{\delta} a_{d} g(\delta) \\
& =\sum_{\delta \leq r} \frac{1}{\delta} g(\delta) A_{F}\left(\frac{r}{\delta}\right)
\end{aligned}
$$

on writing $k=d \delta$ and then summing over $d \leq r / \delta$ first. Substituting from Lemma 3.1, we transform (3.29) into

$$
\begin{aligned}
& B_{F}(r)=\sum_{\delta \leq r} \frac{1}{\delta} g(\delta) \sum_{d \leq r / \delta} \frac{\mu(d)}{d} \int_{1}^{r / \delta} \frac{I\left(\frac{t}{d}\right)}{t} G\left(\frac{\delta t}{r}\right) \mathrm{d} t \\
& =\sum_{n \leq r} \frac{1}{n} \sum_{d \mid n} \mu\left(\frac{n}{d}\right) g(d) \int_{n / d}^{r / d} \frac{I\left(\frac{u d}{n}\right)}{u} G\left(\frac{u d}{r}\right) \mathrm{d} u
\end{aligned}
$$

after writing $n=d \delta$, eliminating $\delta$ as $\frac{n}{d}$ and making the change of variables $\frac{t}{d}=\frac{n}{d} u$.

Further, writing $u d=v$, the integral becomes $\int_{n}^{r / d} \frac{I\left(\frac{v}{n}\right)}{v} G\left(\frac{v}{r}\right) \mathrm{d} v$ since $I(x)=0$ for $x<1$ and indeed, the integral is independent of $d$. Hence 


$$
B_{F}(r)=\sum_{n \leq r} \frac{h(n)}{n} \int_{1}^{r} \frac{I\left(\frac{v}{n}\right)}{v} G\left(\frac{v}{r}\right) \mathrm{d} v
$$

or $(3.26)$.

To deduce (3.27), we put $t=\frac{r n}{w}$ in (3.26) and note that the resulting integral over $n \rightarrow r n$ amounts to $1 \rightarrow r$ in view of the vanishing properties of $I$ and $G$. The result follows by changing the order of summation and integration, completing the proof.

By (3.19), (3.28) reads

$$
H_{h}(w)=\sum_{n \leq w} \frac{h(n)}{n} F\left(\frac{n}{w}\right)-\frac{n}{w} \sum_{n \leq w} \frac{h(n)}{n} F^{\prime}\left(\frac{n}{w}\right)
$$

in which the first term on the right may be expressed as

$$
\sum_{n \leq w} \frac{h(n)}{n} F\left(\frac{n}{w}\right)=\int_{w}^{\infty} \frac{1}{u^{2}} \sum_{n \leq u} h(n) F^{\prime}\left(\frac{n}{u}\right) \mathrm{d} u
$$

on writing

$$
F\left(\frac{n}{w}\right)=-\int_{w}^{\infty} \frac{\mathrm{d}}{\mathrm{d} u} F\left(\frac{n}{u}\right) \mathrm{d} u=\int_{w}^{\infty} F^{\prime}\left(\frac{n}{u}\right) \frac{n}{u^{2}} \mathrm{~d} u .
$$

Hence integrating $(3.32)$ over $[2, \infty)$ and incorporating $(3.33)$ we deduce that

$$
\int_{2}^{\infty} \frac{|H(w)|}{w} \mathrm{~d} w \leq \int_{2}^{\infty} \frac{|J(w)|}{w} \mathrm{~d} w+|J(2)|,
$$

where

$$
J(x)=\int_{x}^{\infty} \frac{1}{w^{2}} K(w) \mathrm{d} w
$$

and where

$$
K(w)=\sum_{n \leq w} h(n) F^{\prime}\left(\frac{n}{w}\right) .
$$

The main task from here is to establish the following

Lemma 3.3. We have

$$
\int_{2}^{\infty} \frac{|H(w)|}{w} \mathrm{~d} w=O(1) .
$$

Proof. We estimate the functions $K, J$ and finally $\int_{2}^{\infty} \frac{|H(w)|}{w} \mathrm{~d} w$ in (3.35).

To estimate $K(w)$ we divide it into two: $\Sigma_{1}, \Sigma_{2}$, where the sum is over $n \leq \alpha$ and $\alpha \leq n \leq w$, respectively, where $\alpha=\alpha(w)$ is chosen later to be near to $w$ up to a small distance of the order of $\delta$. By partial summation and (1.6), we obtain

$$
\left|\Sigma_{1}\right|=\sum_{n \leq \alpha} \ll \alpha \delta(\alpha) F^{\prime}\left(\frac{\alpha}{w}\right),
$$

which is the hardest term in Segal's treatment.

For $\Sigma_{2}$ we find a formula by integration by parts

$$
\begin{aligned}
& \int_{n-1}^{n}(t-[t]) \mathrm{d} F^{\prime}\left(\frac{t}{w}\right)=\left[(t-[t]) F^{\prime}\left(\frac{t}{w}\right)\right]_{n-1}^{n}-\int_{n-1}^{n} F^{\prime}\left(\frac{t}{w}\right) \mathrm{d}(t-[t]) \\
& =F^{\prime}\left(\frac{n}{w}\right)-\int_{n-1}^{n} F^{\prime}\left(\frac{t}{w}\right) \mathrm{d} t .
\end{aligned}
$$


Since the left-hand side is $\int_{n-1}^{n}(t-[t]) \frac{1}{w} F^{\prime \prime}\left(\frac{t}{w}\right) \mathrm{d} t$, it follows that

$$
F^{\prime}\left(\frac{n}{w}\right)=\int_{n-1}^{n} F^{\prime}\left(\frac{t}{w}\right) \mathrm{d} t+\int_{n-1}^{n}(t-[t]) \frac{1}{w} F^{\prime \prime}\left(\frac{t}{w}\right) \mathrm{d} t .
$$

Summing (3.41) over $([\alpha],[w])$ and using properties of $-F^{\prime}$ and (1.7) we deduce that

$$
\begin{aligned}
& (\log w)^{-A}\left|\Sigma_{2}\right| \leq \sum_{\alpha \leq n \leq w}-F^{\prime}\left(\frac{n}{w}\right) \\
& \ll-w F\left(\frac{[w]}{w}\right)+w F\left(\frac{[\alpha]}{w}\right)-F^{\prime}\left(\frac{[w]}{w}\right)+F^{\prime}\left(\frac{[\alpha]}{w}\right) \\
& \ll w F\left(\frac{[\alpha]}{w}\right)-F^{\prime}\left(\frac{[w]}{w}\right)
\end{aligned}
$$

by the properties of $F$. Hence together with (3.39) this implies

$$
|K(w)| \ll-\alpha \delta(\alpha) F^{\prime}\left(\frac{\alpha}{w}\right)+(\log w)^{A}\left(w F\left(\frac{[\alpha]}{w}\right)-F^{\prime}\left(\frac{[w]}{w}\right)\right)
$$

which amounts to

$$
|K(w)| \ll-w \delta(w) F^{\prime}(1-\delta(w))+(\log w)^{A}\left(w F(1-\delta(w))-F^{\prime}\left(\frac{[w]}{w}\right)\right)
$$

on choosing

$$
\alpha(w)=1-\delta(w)
$$

Substituting (3.43) in (3.36), we obtain

$$
\begin{aligned}
|J(x)| & \ll-\int_{x}^{\infty} \frac{1}{w} \delta(w) F^{\prime}(1-\delta(w)) \mathrm{d} w+\int_{x}^{\infty} \frac{(\log w)^{A}}{w} F(1-\delta(w)) \mathrm{d} w \\
& -\int_{x}^{\infty} \frac{(\log w)^{A}}{w^{2}} F^{\prime}\left(\frac{[w]}{w}\right) \mathrm{d} w
\end{aligned}
$$

Recalling the form of $\delta$ in (1.4), we see that $\delta^{\prime}$ is of the form $\frac{1}{w}(\log w)^{c} \delta(w)=\frac{1}{w} \delta(w)$. Hence the first term on the right of $(3.45)$ is $[\delta(w) F(1-\delta(w))]_{x}^{\infty}=\delta(x)$ by (1.3). The mid term is of the same form by (1.3). For the last term we express it as

$$
-\sum_{n \geq x} \int_{n}^{n+1} \frac{1}{w^{2-\epsilon}} F^{\prime}\left(\frac{n}{w}\right) \mathrm{d} w=\sum_{n \geq x} \frac{1}{n^{1-\epsilon}} F\left(\frac{n}{n+1}\right) \ll_{A} \sum_{n \geq x} \frac{1}{(\log n)^{A}} F\left(1-\frac{1}{n+1}\right) .
$$

Hence by (1.3), the third term is

$$
\int_{x}^{\infty} \frac{1}{t} \delta(t) \mathrm{d} t=\delta(x)
$$

Hence substituting the previous results in (3.35), we obtain (3.38).

We are now in a position to prove

Theorem 3.4. We suppose all the assumptions in Assumption are satisfied. If $\sum a_{n}$ is I-summable to $A$, then $\sum b_{n}$ (with $b_{n}$ defined in (3.25)) is $F$-summable to $A$ for any $F \in \mathcal{F}$. 
Proof. In (3.27) we divide the integral over $[1, r]$ into $[1, \sqrt{r}] \cup[\sqrt{r}, r]$ and substitute the result $I(x)=$ $A+o(1)$ for $r / w \geq \sqrt{r}$ and $I(x)=o(1)$ into the respective integrals, we deduce that

$$
B_{F}(r)=A \int_{1}^{\sqrt{r}} \frac{H(w)}{w} \mathrm{~d} w+o(1)
$$

as $r \rightarrow \infty$ since the integral is absolutely convergent in view of Lemma 3.3.

On determining the value of the infinite integral as in [Se75, p.135], we complete the proof.

Remark 3.5. (3.27) is the formula appearing on [Se75, p. 137] and is a generalization of [Se75, Formula (6)], which is (3.18). This follows on choosing $g$ in Proposition 3.2 to be the multiplicative identity, i.e. $g(1)=1$ and $g(n)=0$ for $n \neq 1$. Hence Theorem 3.4 entails [Se 75, Theorem 1] to the effect that I-summability implies F-summability.

\section{References}

[Ap57] T. M. Apostol, Mathematical analysis, Addison-Wesley Publishing Company, Inc., Reading, Mass., 1957.

[CM52] K. Chandrasekharan and S. Minakshisundaram, Typical means, OUP, Oxford 1952.

[Da80] H. Davenport, Multiplicative number theory, 1st ed. Markham, Chicago 1967, 2nd ed. Springer, New York etc. 1980.

[deH70] L. de Haan, On regular variation and its applications to the weak convergence of sample extremes, Math. Center Tracts 32, Amsterdam 1970.

[Har49] G. H. Hardy, Divergent series, OUP, Oxford 1949.

[HR72] G. H. Hardy and M. Riesz, The general theory of Dirichlet's series, CUP, Cambridge 1915; reprint, Hafner, New York 1972 .

[HW15] G. H. Hardy and E. M. Wright, Introduction to the theory of numbers, OUP, Oxford 1915.

[In45] A. E. Ingham, Some tauberian theormes connected with the prime number theorem, J. London Math. Soc. 20 (1945), 171-180-142.

[Iv85] A. Ivić, The Riemann Zeta-Function, John Wiley \& Sons, New York, 1985.

[Se75] S. L. Segal, Ingham's summability and Riemann's hypothesis, Proc. London Math. Soc. (3) 30 (1975), 139-142.

[Sen76] E. Seneta, Regularly varying functions, Lect. Notes Math. 508, Springer Verl., Berlin-Heidelberg-New York 1976.

[Tit86] E. C. Titchmarsh, The Theory of the Riemann Zeta-Function, (second edition revised by D. R. Heath-Brown), OUP, Oxford 1986.

[Wi89] D. W. Widder, Advanced calculus, 2nd ed. Dover Publ., New York 1989.

[Win43] A. Wintner, Eratosthenian averages, Wavery Press, New York 1943.

[Win57] A. Wintner, On arithmetical summation processes, Amer. J. Math. 79 (1957), 551-574.

\section{Shigeru Kanemitsu}

Graduate School of Engrg

Kyushu Inst. Tech.

1-1 Sensuicho Tobata

Kitakyushu 804-8555, Japan

e-mail: omnikanemitsu@yahoo.com

\section{Takako Kuzumaki}

Faculty of Engineering

Gifu University

Gifu 501-1193, Japan

e-mail: kuzumaki@gifu-u.ac.jp

\section{Yoshio Tanigawa}

Graduate School of Mathematics

Nagoya University

Furo-cho, Nagoya 464-8602, Japan

e-mail: : tanigawa@math.nagoya-u.ac.jp 